\title{
Some matrix elements for Morse oscillators*
}

\author{
Jason A. C. Gallas ${ }^{\dagger}$ \\ Instituto de Física, Universidade Federal do Rio Grande do Sul, 90000 Porto Alegre, R. S., Brazil
}

(Received 3 October 1979)

\begin{abstract}
In this paper general working equations for the Morse $\left(r-r_{e}\right)^{l}$ matrix elements are given. These equations can be used to calculate the diagonal $(m=n)$ matrix elements and, for the off-diagonal $(m \neq n)$ elements, are simpler to use than the ones currently available in the literature. Also, in this paper a new approach is given which allows one to obtain simple formulas, in closed form, for the off-diagonal matrix elements. Explicit expressions are given for $l=1,2$, and 3 .
\end{abstract}

\section{INTRODUCTION}

Fifty years ago Morse ${ }^{1}$ proposed the potential

$$
V(r)=D e^{-2 a\left(r-r_{e}\right)}-2 D e^{-a\left(r-r_{e}\right)}
$$

as a model to describe the electronic levels of diatomic molecules. Since then this potential, known as the Morse potential, has been used in the study of diatomic molecules. Because it describes the two-atomic molecular vibrations excellently, ${ }^{2}$ it has been extensively used to calculate vibrational transition probabilities, to predict band intensities, oscillator strengths, and related parameters. ${ }^{3}$ Recently, this same potential has been found to be useful in the study of molecular dissociation under intense electromagnetic fields ${ }^{4}$ and, more generally, in the study of the interaction of coherent radiation with molecules. ${ }^{5}$ In all these applications of the Morse potential the evaluation of several matrix elements is needed. In the majority, these matrix elements are of the general type

$$
M_{m n}^{(l)}=\left\langle m\left|\left(r-r_{e}\right)^{l}\right| n\right\rangle, \quad l=0,1,2,3, \ldots .
$$

For vibrational transitions involving two different electronic states, with each state represented by a potential given by Eq. (1), the calculation of the matrix elements is usually done by direct numerical integration or by an approximate method known as the $\alpha$-average method. ${ }^{3,6}$ Recently, an analytical expression has been derived ${ }^{7,8}$ which enables one to easily evaluate such matrix elements without any approximation. These general expressions also include the effects of vibrationrotation interaction.

For transitions within the same electronic state, i.e., within the same Morse state, one can find in the literature ${ }^{9}$ expressions for the $l=1$ and $l=2$ matrix elements of Eq. (2) when $m \neq n$. Some calculations have also included the effects of rotation. ${ }^{10}$. In particular, Herman and Rubin ${ }^{11}$ have given general expressions for the matrix elements of a rotating Morse oscillator for any desired $l$ value in Eq. (2). However, as noted by Cashion, ${ }^{10}$ these expressions are sufficiently complex to deter one from using them. Also, since all of the previous work was in the calculation of the vibrational transition probabilities for diatomic molecules, the expressions available in the literature $\mathrm{r}^{10,12}$ are given for the off-diagonal $(m \neq n)$ matrix elements.

In the present paper we derive general working equations to calculate the matrix elements of Eq. (2), for transitions within a given Morse state, for all $m$ and $n$, and, in principle, for all $l$ values, too. The diagonal matrix elements $(m=n)$, which are very important in the theoretical investigation of the interaction of coherent radiation with molecules as recently reported by Nieto and Simmons, ${ }^{5}$ as far as the author knows, have not been reported before for vibrational states other than the ground state. Also, in this paper a new way to calculate the off-diagonal matrix elements, which allows one to derive simpler working equations for these elements, is given.

\section{RESULTS AND DISCUSSION}

Strictly speaking, the Schrödinger equation for the Morse potential cannot be exactly solved. ${ }^{13}$ However, to a very good approximation one can consider this equation as being solvable for diatomic molecules. ${ }^{2,14}$ In this case the following orthonormalized eigenkets are obtained ${ }^{14}$ :

$$
\langle r \mid n\rangle=N_{n} e^{-z^{\prime 2}} z^{b / 2} L_{n}^{b}(z),
$$

where

$$
\begin{aligned}
& N_{n}=\left(\frac{a b n !}{\Gamma(k-n)}\right)^{1 / 2}, \\
& z=k \exp \left[-a\left(r-r_{e}\right)\right], \\
& b=k-2 n-1,
\end{aligned}
$$

and where, for a diatomic molecule of reduced mass $\mu$ and spectroscopic constants ${ }^{15} \omega_{e}, \omega_{e} x_{e}$, and $B_{e}$,

$$
k=\omega_{e} / \omega_{e} x_{e},
$$




$$
\begin{aligned}
& r_{e}=1 /\left[0.243559\left(\mu B_{e}\right)^{1 / 2}\right], \\
& a=0.243559\left(\mu w_{e} x_{e}\right)^{1 / 2}, \\
& D=w_{e}^{2} /\left(4 w_{e} x_{e}\right) .
\end{aligned}
$$

The Laguerre polynomials in Eq. (3) are either given by ${ }^{16}$

$$
L_{n}^{b}(z)=\sum_{i=0}^{n}\left(\begin{array}{c}
n+b \\
n-i
\end{array}\right) \frac{(-z)^{i}}{i !}
$$

or by the formula

$$
L_{n}^{b}(z)=\frac{1}{n ! e^{-z} z^{b}} \frac{d^{n}}{d z^{n}}\left(e^{-z} z^{n+b}\right) .
$$

To calculate the linear $\left\langle m\left|r-v_{e}\right| n\right\rangle$ matrix we have to solve the integral

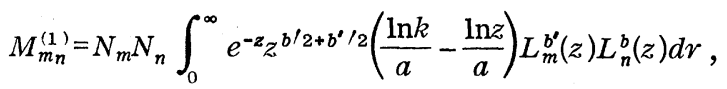

where $b^{\prime}=k-2 m-1$. From Eq. (5) one easily finds $d r=-d z /(a z)$. With this, Eq. (13) can also be written as

$$
\begin{aligned}
M_{m_{n}}^{(1)}=\frac{N_{m} N_{n}}{a} \int_{0}^{k e e^{a r} e} & e^{-z} z^{b / 2+\delta / 2-1} \\
& \times\left(\frac{\ln k}{a}-\frac{\ln z}{a}\right) L_{m}^{b^{\prime}}(z) L_{n}^{b}(z) d z .
\end{aligned}
$$

Following ter Haar, ${ }^{13}$ we replace $k e^{a r_{e}}$ by infinity in Eq. (14) since the error introduced is negligible. Noting that the eigenkets are orthonormalized, we obtain

$$
M_{m n}^{(1)}=\frac{\ln k}{a} \delta_{m, n}+\frac{N_{m} N_{n}}{a} \int_{0}^{\infty} e^{-z} z^{b / 2+b^{\prime} / 2-1}\left(-\frac{\ln z}{a}\right) L_{m}^{b^{\prime}}(z) L_{n}^{b}(z) d z
$$

Next we use the definition of the Laguerre polynomials Eq. (11) twice in Eq. (15) to find

$$
M_{m n}^{(1)}=\frac{\ln k}{a} \delta_{m, n}+\frac{N_{m} N_{n}}{a^{2}} \sum_{i=0}^{m} \sum_{j=0}^{n} \frac{(-1)^{i+j+1}}{i ! j !}\left(\begin{array}{c}
m+b^{\prime} \\
m-i
\end{array}\right)\left(\begin{array}{c}
n+b \\
n-j
\end{array}\right) \int_{0}^{\infty} e^{-z} z^{b / 2+b^{\prime} / 2+i+j-1} \ln z d z .
$$

The integral appearing in this expression is evaluated [Eq. (4.352-1) of Ref. 16] and finally the linear matrix elements are given by

$$
M_{m n}^{(1)}=\frac{\ln k}{a} \delta_{m, n}+\frac{N_{m} N_{n}}{a^{2}} \sum_{i=0}^{m} \sum_{j=0}^{n} \frac{(-1)^{i+j+1}}{i ! j !}\left(\begin{array}{c}
m+b^{\prime} \\
m-i
\end{array}\right)\left(\begin{array}{c}
n+b \\
n-j
\end{array}\right) \Gamma(k+i+j-n-m-1) \psi(k+i+j-n-m-1),
$$

where $\psi(x)=(d / d x)[\ln \Gamma(x)]$ is the digamma function (Ref. 16, p. 943). From this result the expectation value $\langle x\rangle_{0}$ in Eq. (5.15) of Ref. 7 can be trivially obtained by setting $m=n=0$.

For $m \neq n$, a new approach can be used to calculate the linear matrix elements. This new approach consists of replacing one of the Laguerre polynomials in Eq. (15) by Eq. (11) and the other one by Eq. (12) and then performing several integrations by parts. Since $M_{m n}^{(l)}=M_{n m}^{(l)}$ we assume $n>m$ for convenience. After substitution of the Laguerre polynomials, Eq. (15) becomes

$$
\begin{aligned}
M_{m n}^{(1)}=\frac{N_{m} N_{n}}{a^{2}} \frac{1}{n !} \sum_{i=0}^{m} & \left(\begin{array}{c}
m+b^{\prime} \\
m-i
\end{array}\right) \frac{(-1)^{i+1}}{i !} \\
& \times \int_{0}^{\infty} \ln z z^{p} \frac{d^{n}}{d z^{n}}\left(e^{-z} z^{n+b}\right) d z,
\end{aligned}
$$

where the power $p$ of $z$ is given by

$$
p=\frac{1}{2} b+\frac{1}{2} b^{\prime}-b+i-1=n+i-m-1 \text {. }
$$

With these substitutions the problem of calculating $\left\langle m\left|r-r_{e}\right| n\right\rangle$ reduces to the evaluation of the integral

$$
I_{1}=\int_{0}^{\infty} \ln z z^{p} \frac{d^{n}}{d z^{n}}\left(e^{-z} z^{n+b}\right) d z, \quad n>p .
$$

Now, due to the vanishing of $e^{-z} z^{n+b}$ at the limits of integration, it is easy to evaluate Eq. (20) by means of $n$ integrations by parts. This can be done in two steps: First, in order to remove the $z^{p}$ term in the integral, we integrate $p$ times by parts; then the integral is reduced to a known one by performing the remaining $n-p$ integrations. After $p$ integrations by parts, the nonvanishing contribution is given by

$$
I_{1}=(-1)^{p} p ! \int_{0}^{\infty} \ln z \frac{d^{n-p}}{d z^{n-p}}\left(e^{-z} z^{n+b}\right) d z .
$$

Integrating by parts $(n-p)$ times one obtains

$$
\begin{aligned}
I_{1} & =(-1)^{p} p !\left(-(m-i) ! \int_{0}^{\infty} e^{-z} z^{k+i-n-m-2} d z\right) \\
& =(-1)^{p+1} p !(m-i) ! \Gamma(k+i-n-m-1) .
\end{aligned}
$$

With this result Eq. (18) then becomes

$$
M_{m n}^{(1)} \frac{N_{m} N_{n}}{a^{2}} \frac{\Gamma(k-m)}{n !}(-1)^{n-m+1} \sum_{i=0}^{m} \frac{(n+i-m-1) ! \Gamma(k+i-n-m-1)}{i ! \Gamma(k+i-2 m)}, \quad n>m .
$$


This equation can be further simplified if we note that for $n>m$

$$
\begin{aligned}
\frac{m !}{n !} \frac{\Gamma(k-m)}{\Gamma(k-n)} \sum_{i=0}^{m} \frac{(n+i-m-1) ! \Gamma(k+i-n-m-1)}{i ! \Gamma(k+i-2 m)} \\
=\frac{1}{(n-m)(k-n-m-1)}
\end{aligned}
$$

Substitution of this result in Eq. (23) gives

$$
\begin{gathered}
M_{m n}^{(1)}=\frac{(-1)^{n-m+1}}{a(n-m)(k-n-m-1)}\left(\frac{n !}{m !} \frac{\Gamma(k-n)}{\Gamma(k-m)} b b^{\prime}\right)^{1 / 2}, \\
n>m,
\end{gathered}
$$

which is a relatively simple expression for the off-diagonal linear matrix elements. This expression can be easily generalized to include rotational effects, since Pekeris ${ }^{17}$ has shown that these effects only introduce a slight $J$ (rotational quantum number) dependence in $k .^{7,17}$

In studying the harmonic band of hydrogen chloride, Dunham ${ }^{18}$ calculated the matrix element $\left\langle 0\left|r-r_{e}\right| 2\right\rangle$ :

$$
\begin{aligned}
M_{02}^{(1)}=p_{0}^{\prime} A_{0} A_{2} k^{k-3}[ & k^{2} J_{k-1}-2 k(k-3) J_{k-2} \\
& \left.+(k-3)(k-4) J_{k-3}\right],
\end{aligned}
$$

where all the symbols in the right-hand side are defined in the original paper. ${ }^{18}$ After some simplifications and algebraic manipulation, this equa- tion can be written as

$$
\begin{aligned}
M_{02}^{(1)} & =-\frac{1}{a}\left(\frac{k-5}{2(k-2)}\right)^{1 / 2} \\
& \times[(k-4) \psi(k-3)-2(k-3) \psi(k-2)+(k-2) \psi(k-1)],
\end{aligned}
$$

which is the result given in Eq. (17). This matrix element can be further simplified if one notes that

$$
\begin{array}{r}
{[(k-4) \psi(k-3)-2(k-3) \psi(k-2)+(k-2) \psi(k-1)} \\
=1 /(k-3) .
\end{array}
$$

Finally,

$$
M_{02}^{(1)}=-\frac{1}{a}\left(\frac{k-5}{2(k-2)}\right)^{1 / 2} \frac{1}{k-3} .
$$

With this simple result, which can be directly obtained from Eq. (25), the intensity of the harmonic band for any molecule can be calculated with a pocket calculator.

For the higher-order matrix elements, the calculations are similar. Noting that

$$
\left(r-r_{e}\right)^{l}=\left(\frac{\ln k}{a}-\frac{\ln z}{a}\right)^{l}
$$

according to Eq. (5), the second-order $(l=2)$ matrix element, for any integer value of $m$ and $n$, is given by

$$
M_{m n}^{(2)}=\frac{N_{m} N_{n}}{a} \sum_{i=0}^{m} \sum_{j=0}^{n} \frac{(-1)^{i+j}}{i ! j !}\left(\begin{array}{c}
m+b^{\prime} \\
m-i
\end{array}\right)\left(\begin{array}{c}
n+b \\
n-j
\end{array}\right) \int_{0}^{\infty} e^{-z} z^{b / 2+b^{\prime} / 2+i+j-1}\left(\frac{\ln k}{a}-\frac{\ln z}{a}\right)^{2} d z
$$

Now, by changing variable of integration from $z$ to $y=z / k$ and using the results from the Appendix, we find

$$
\begin{aligned}
M_{m n}^{(2)}=\frac{N_{m} N_{n}}{a^{3}} \sum_{i=0}^{m} \sum_{j=0}^{n} & \frac{(-1)^{i+j}}{i ! j !}\left(\begin{array}{c}
m+b^{\prime} \\
m-i
\end{array}\right)\left(\begin{array}{c}
n+b \\
n-j
\end{array}\right) \Gamma(k+i+j-n-m-1) \\
& \times\left\{[\psi(k+i+j-n-m-1)-\ln k]^{2}+\psi^{(1)}(k+i+j-n-m-1)\right\} .
\end{aligned}
$$

For $m=n=0$ this result reduces to Eq. (5.17) of Ref. 7. The second-order off-diagonal matrix elements can also be evaluated in a simple way by using a suitable representation for the Laguerre polynomials in the following equation:

$$
M_{m n}^{(2)}=\frac{2 \ln k}{a} M_{m n}^{(1)}+\frac{N_{m} N_{n}}{a} \int_{0}^{\infty} e^{-z} z^{b / 2+b^{\prime} / 2-1}\left(\frac{\ln z}{a}\right)^{2} L_{m}^{b^{\prime}}(z) L_{n}^{b}(z) d z, \quad n>m .
$$

Substitution of Eq. (11) and Eq. (12) into Eq. (33) gives

$$
M_{m n}^{(2)}=\frac{2 \ln k}{a} M_{m n}^{(1)}+\frac{N_{m} N_{n}}{a^{3}} \frac{1}{n !} \sum_{i=0}^{m} \frac{(-1)^{i}}{i !}\left(\begin{array}{c}
m+b^{\prime} \\
m-1
\end{array}\right) \int_{0}^{\infty}(\ln z)^{2} z^{p} \frac{d^{n}}{d z^{n}}\left(e^{-z} z^{n+b}\right) d z, \quad n>m
$$

where $p$ is given by Eq. (19). To calculate this matrix element we need to evaluate the integral

$$
I_{2}=\int_{0}^{\infty}(\ln z)^{2} z^{p} \frac{d^{n}}{d z^{n}}\left(e^{-z} z^{n+b}\right) d z, \quad n>p .
$$

This integral can be easily evaluated in the same way as $I_{1}$ was in Eq. (20). After $p$ integrations by parts, one gets 


$$
I_{2}=(-1)^{p} p !\left(\int_{0}^{\infty}(\ln z)^{2} \frac{d^{n-p}}{d z^{n-p}}\left(e^{-z} z^{n+b}\right) d z+2[\psi(p+1)+\gamma] \int_{0}^{\infty} \ln z \frac{d^{n-p}}{d z^{n-p}}\left(e^{-z} z^{n+b}\right) d z\right)
$$

where $\gamma=0.5577215 \cdots$ is the Euler constant. Performing the remaining $n-p+1$ integ rations in the first integral of Eq. (36) we find

$$
\int_{0}^{\infty}(\ln z)^{2} \frac{d^{n-p}}{d z^{n-p}}\left(e^{-z} z^{n+b}\right) d z=2(m-i) ! \Gamma(k+i-n-m-1)[\psi(m-i+1)-\psi(k+i-n-m+1)+\gamma] .
$$

Then substituting Eq. (22) and these results back into Eq. (34) the second-order matrix element becomes

$$
\begin{aligned}
M_{m n}^{(2)}=\frac{2 \ln k}{a} M_{m n}^{(1)}+ & \frac{2}{a^{2}}(-1)^{n-m-1}\left(\frac{m !}{n !} \frac{\Gamma(k-m)}{\Gamma(k-n)} b b^{\prime}\right)^{1 / 2} \\
& \times \sum_{i=0}^{m} \frac{\Gamma(k+i-n-m-1)}{\Gamma(k+i-2 m)} \frac{(n+i-m-1) !}{i !} \\
& \quad \times[\psi(m-i+1)-\psi(n+i-m)-\psi(k+i-n-m-1)] \text { for } n>m .
\end{aligned}
$$

For the off-diagonal matrix elements this last expression is simpler to evaluate then the one given by Eq. (32) since it involves only one summation and two special functions to be evaluated.

For $l=3$, according to Eq. (A5) of the Appendix, we find

$$
\begin{aligned}
M_{m n}^{(3)}=\frac{N_{m} N_{n}}{a^{4}} \sum_{i=0}^{m} \sum_{j=0}^{n} & \frac{(-1)^{i+j+1}}{i ! j !}\left(\begin{array}{c}
m+b^{\prime} \\
m-i
\end{array}\right)\left(\begin{array}{c}
n+b \\
n-j
\end{array}\right) \Gamma(q) \\
& \times\left\{[\psi(q)-\ln k]^{3}+3[\psi(q)-\ln k] \psi^{(1)}(q)+\psi^{(2)}(q)\right\},
\end{aligned}
$$

where $q=k+i+j-n-m-1$. For $n>m$,

$$
M_{m n}^{(3)}=-3\left(\frac{\ln k}{a}\right)^{2} M_{m n}^{(1)}+\frac{3 \ln k}{a} M_{m n}^{(2)}+\frac{N_{m} N_{n}}{a^{4}} \frac{1}{n !} \sum_{i=0}^{m} \frac{(-1)^{i+1}}{i !}\left(\frac{m+b^{\prime}}{m-i}\right) I_{3},
$$

where

$$
I_{3}=\int_{0}^{\infty}(\ln z)^{3} z^{p} \frac{d_{n}}{d z^{n}}\left(e^{-z} z^{n+b}\right) d z,
$$

and $p$ is given by Eq. (19). Integrating Eq. (41) by parts $p$ times the nonvanishing contribution is given by

$$
\begin{aligned}
I_{3}=(-1)^{p} p ! & \left(\int_{0}^{\infty}(\ln z)^{3} \frac{d^{n-p}}{d z^{n-p}}\left(e^{-z} z^{n+b}\right) d z+3[\psi(p+1)+\gamma] \int_{0}^{\infty}(\ln z)^{2} \frac{d^{n-p}}{d z^{n-p}}\left(e^{-z} z^{n+b}\right) d z\right. \\
& \left.+6\left(1-\delta_{0, p}\right)\left(1-\delta_{1, p}\right) S_{p} \int_{0}^{\infty} \ln z \frac{d^{n-p}}{d z^{n-p}}\left(e^{-z} z^{n+b}\right) d z\right),
\end{aligned}
$$

where

$$
S_{p}=\sum_{j=0}^{p-1} \frac{1}{p-j}[\psi(p+1)-\psi(p+1-j)] \text { for } p \geqslant 2 \text { and } S_{0}=S_{1}=0 .
$$

In Eq. (42) the only unknown integral is the first one involving the term $(\ln z)^{3}$ in the integrand. As before, integrating by parts $(n-p)$ times and then using Eq. (A4) from the Appendix, we find

$$
\int_{0}^{\infty}(\ln z)^{3} \frac{d^{n-p}}{d z^{n-p}}\left(e^{-z} z^{n+b}\right) d z=-3(m-i) ! \Gamma(t)\left\{\psi(t)\left[\psi(t)-\left(1-\delta_{1, p}\right)\left(2-Z_{p}\right)\right]+Y_{p}+\psi^{(1)}(t)\right\},
$$

where $t=k+i-n-m-1$ and

$$
\begin{aligned}
Z_{p}=\psi\left(\frac{1}{2} p\right)+\psi\left(\frac{1}{2} p+\frac{1}{2}\right)+2 \gamma+2 \ln 2-2, \\
\qquad \text { for } p \geqslant 3, Z_{1}=Z_{2}=0,
\end{aligned}
$$

$$
\begin{aligned}
Y_{p}=Z_{p}+\left(1-\delta_{3, p}\right)\left[\frac{1}{3}+\right. & \left.X_{p}\left(1-\delta_{4, p}\right)\right] \\
& \text { for } p \geqslant 3, Y_{1}=Y_{2}=0,
\end{aligned}
$$


TABLE I. Numerators of the rational coefficients $S_{p}$, $Z_{p}, Y_{p}$, and $X_{p}$.

\begin{tabular}{rrrrrr}
\hline \hline$p$ & $p !$ & $p ! S_{p}$ & $(\phi-1) ! Z_{p}$ & $(\phi-1) ! Y_{p}$ & $(p-1) ! X_{p}$ \\
\hline 1 & 1 & 0 & 0 & 0 & 0 \\
2 & 2 & 1 & 0 & 0 & 0 \\
3 & 6 & 6 & 2 & 2 & 0 \\
4 & 24 & 35 & 10 & 12 & 0 \\
5 & 120 & 225 & 52 & 70 & 10 \\
6 & 720 & 1624 & 308 & 450 & 102 \\
7 & 5040 & 13132 & 2088 & 3248 & 920 \\
8 & 40320 & 118124 & 16056 & 26264 & 8528 \\
9 & 362880 & 1172700 & 138528 & 236248 & 84280 \\
10 & 3628800 & 12753576 & 1327392 & 2345400 & 897048 \\
\hline \hline
\end{tabular}

$$
X_{p}=\sum_{j=0}^{p-5} \frac{1}{p-1-j} Z_{p-1-j} \text { for } p \geqslant 5 .
$$

It is interesting to note that since $S_{p}, Z_{p}, Y_{p}$ and $X_{p}$ do not depend on the Morse parameters, they need be evaluated just once and then may be used in calculations for any set of Morse parameters, i.e., for any molecule. The first ten values of $S_{p}$, $Z_{p}, Y_{p}$, and $X_{p}$ are given in Table $\mathrm{I}$.

In principle, for any $l$ value, the above procedures can be repeated to give the corresponding $M_{m n}^{(l)}$ matrix elements, but as $l$ increases, the complexity of the $M_{m n}^{(l)}$ also increases. However, the approach presented here gives results for the diagonal matrix elements as well as for the offdiagonal elements. The expressions obtained for the off-diagonal matrix elements are simpler to evaluate then the others found in the literature, e.g., the ones given by Hermann and Rubin. ${ }^{11}$ Hopefully, one may find relations like Eq. (24), which enable the expressions for the higher-order matrix elements to be further simplified.

It is worth mentioning that even though the present derivation does not include the effects of vibration-rotation interaction, this can be easily done in view of the results of Pekeris ${ }^{17}$.

\section{CONCLUSIONS}

We have derived expressions for the matrix elements of $\left(r-r_{e}\right)^{l}$ for $l=1,2$, and 3 between Morse eigenstates. As far as the author knows, this is the first time that general expressions have been given for the diagonal matrix elements, which are important in the study of the interaction of coherent radiation with molecules, as mentioned in Refs. 4 and 5. For the off-diagonal matrix elements a new method of calculation is presented. This method, which makes use of a special representation of Laguerre polynomials and of sequences of integrations by parts, gives simpler equations for the off-diagonal matrix elements than the ones currently available in the literature. These off-diagonal matrix elements are important in the calculation of the intensity distribution in the vibration-rotation spectrum of diatomic molecules $^{9-12,18}$ as well as in the theoretical investigation of the dipole moment function of diatomics. ${ }^{19}$

\section{ACKNOWLEDGMENTS}

The author thanks Dr. V. E. Herscovitz and Dr. J. D. Gaffey, Jr. for critically reading the manuscript. This work was partially supported by the Brazilian agencies CNPq and FINEP.

\section{APPENDIX}

The integrals 4.358-2 and 4.358-3 in the table of Gradshteyn and Ryzhik ${ }^{16}$ are incorrect. With the notation of these authors they should read

$$
\int_{0}^{\infty} x^{\nu-1} e^{-\mu x}(\ln x)^{2} d x=\frac{\Gamma(\nu)}{\mu^{\nu}}\left\{[\psi(\nu)-\ln \mu]^{2}+\zeta(2, \nu)\right\}, \quad \operatorname{Re} \mu>0, \operatorname{Re} \nu>0
$$

and

$$
\int_{0}^{\infty} x^{\nu-1} e^{-\mu x}(\ln x)^{3} d x=\frac{\Gamma(\nu)}{\mu^{\nu}}\left\{[\psi(\nu)-\ln \mu]^{3}+3[\psi(\nu)-\ln \mu] \xi(2, \nu)-2 \zeta(3, \nu)\right\}, \quad \operatorname{Re} \mu>0, \operatorname{Re} \nu>0 .
$$

Using the result

$$
\psi^{(n)}(\nu)=(-1)^{n+1} n ! \zeta(n+1, \nu), \quad \nu \neq 0,-1,-2, \ldots
$$

which can be obtained from Eq. 9.521-1 of Ref. (16) and Eq. 6.4.10 of Ref. (20), these integrals can be more conveniently written as 


$$
\int_{0}^{\infty} x^{\nu-1} e^{-\mu x}(\ln x)^{2} d x=\frac{\Gamma(\nu)}{\mu^{\nu}}\left\{[\psi(\nu)-\ln \mu]^{2}+\psi^{(1)}(\nu)\right\}, \quad \operatorname{Re} \mu>0, \operatorname{Re} \nu>0
$$

and

$$
\int_{0}^{\infty} x^{\nu-1} e^{-\mu x}(\ln x)^{3} d x=\frac{\Gamma(\nu)}{\mu^{\nu}}\left\{[\psi(\nu)-\ln \mu]^{3}+3[\psi(\nu)-\ln \mu] \psi^{(1)}(\nu)+\psi^{(2)}(\nu)\right\}, \quad \operatorname{Re} \mu>0, \quad \operatorname{Re} \nu>0 .
$$

*Part of the paper was presented in the 31st Annual Meeting of the Brazilian Society for the Progress of Science, Fortaleza, July 11-18, 1979.

$\dagger$ Present address: Univer sidade Federal de Santa Catarina, Departamento de Física-Campus da Trindade, 88000 Florianópolis, S. C., Brasil.

1P. M. Morse, Phys. Rev. 34, 57 (1929).

${ }^{2}$ S. Flügge, Practical Quantum Mechanics, (Springer, Berlin, 1974), pp. 182-189.

${ }^{3}$ L. A. Kuznetsova, N. E. Kuzmenko, Yu. Ya. Kuziakov, and Yu. A. Plastinin, Usp. Fiz. Nauk. 113, 285 (1974) [Sov. Phys. Usp. 17, 405 (1974)].

${ }^{4}$ R. B. Walker and R. K. Preston, J. Chem. Phys. 67, 2017 (1977).

${ }^{5}$ M. M. Nieto and L. M. Simmons, Jr., Phys. Rev. A 19,438 (1979).

${ }^{6}$ P. A. Fraser and W. R. Jarmain, Proc. Phys. Soc. London 66A, 1145, (1953); 66A, 1153 (1953).

${ }^{7} \mathrm{~J}$. A. C. Gallas, H. P. Grieneisen, and B. P. Chakraborty, J. Chem. Phys. 69, 612 (1978).

${ }^{8} \mathrm{~J}$. A. C. Gallas, R. E. Francke, H. P. Grieneisen, and B. P. Chakraborty, Astrophys. J., 229, 851
(1979).

${ }^{9}$ H. S. Heaps and G. Herzberg, Z. Phys. 133, 48 (1952).

${ }^{10} \mathrm{~K}$. Cashion, J. Mol. Spectrosc. 10, 182 (1963).

${ }^{11}$ R. Herman and R. J. Rubin, Astrophys. J. 121, 533 (1955).

${ }^{12}$ M. Badawi, N. Bessis, G. Bessis, and G. Hadinger, Can. J. Phys. 52, 110 (1974).

${ }^{13}$ D. ter Haar, Phys. Rev. 70, 222 (1946).

${ }^{14} \mathrm{~J}$. Rundgren, Ark. Fys. 30, 61 (1965).

${ }^{15} \mathrm{G}$. Her zberg, Molecular Spectra and Molecular Structures: I. Spectra of Diatomic Molecules, 2nd. ed. (Van Nostrand, Princeton, 1950).

${ }^{16}$ I. S. Gradshteyn, and I. M. Ryzhik, Tables of Integrals, Series, and Products, 4th. ed., edited by

A. Jeffrey (Academic, New York, 1966).

${ }^{17}$ C. L. Pekeris, Phys. Rev. 45, 98 (1934).

${ }^{18} \mathrm{~J}$. L. Dunham, Phys. Rev. $\underline{35}, 1347$ (1930).

${ }^{19}$ B. S. Rao, J. Phys. B $\underline{4}, 7 \overline{91}$ (1971).

${ }^{20}$ M. Abramowitz and I. A. Stegun, Handbook of Mathematical Functions, National Bureau of Standards Applied Mathematics Series, No. 55 (U.S.G.P.O. Dover, New York, 1965). 